\title{
Large myxomatous odontogenic tumor in the jaw: a case series
}

\author{
Truc Thi Hoang Nguyen*, Mi Young Eo*, Yun Ju Cho, Hoon Myoung, Soung Min Kim \\ Department of Oral and Maxillofacial Surgery, Dental Research Institute, School of Dentistry, Seoul National University, Seoul, Korea
}

\begin{abstract}
J Korean Assoc Oral Maxillofac Surg 2021;47:112-119)
Objectives: Myxomatous odontogenic tumors (MOTs) are the third most common odontogenic tumors in the oral and maxillofacial region. Due to its slow-growing, but locally invasive nature, the tumor is usually detected by accident or only when it becomes a large mass, which causes facial deformity.

Materials and Methods: Current study reports three unusual cases of MOT including huge myxoma involve the mandible in middle-aged man, MOT with ossifying fibroma pattern in mandible, and MOT in maxilla of young female patient. The diagnosis and treatment strategy of MOTs was also summarized and updated.

Results: In reported three cases of patients with large MOTs, surgical treatment was indicated with fibular free flap reconstruction in the mandible and plate reconstruction in the maxilla. The tumors were successfully treated with radical resection and did not show signs of recurrence during the followup period.

Conclusion: Surgical treatment indication depends on size, the position of the lesion, patient systemic condition and surgeon individual experience. In the case of a large tumor, radical resection and reconstruction is the standard surgical strategy. The conservative surgical treatment including enucleation with wide curettage is still under controversy. The recurrence rate for MOTs is significantly high, up to $30 \%$, therefore long-term follow-up is essential.
\end{abstract}

Key words: Odontogenic tumors, Myxoma, Resection margin, Mandibular reconstruction

[paper submitted 2019. 9. 8 / revised 2019. 11. 18 / accepted 2019. 11. 19]

\section{Introduction}

Myxomatous odontogenic tumors (MOTs), including odontogenic myxoma (OM), is the third most common odontogenic tumor after ameloblastoma and odontomas, comprising for $3 \%$ to $20 \%$ of all odontogenic tumors ${ }^{1-4}$. The "odontogenic myxoma" entity was first described by Thoma and Goldman ${ }^{5}$ in 1947. In the World Health Organization (WHO) classification of odontogenic and maxillofacial bone tumors (2017), OM was defined as benign, slow-growing but locally aggressive primordial odontogenic tumors ${ }^{6}$. These

\footnotetext{
Soung Min Kim

Department of Oral and Maxillofacial Surgery, School of Dentistry, Seoul National University, 101 Daehak-ro, Jongno-gu, Seoul 03080, Korea

TEL: +82-2-2072-0213

E-mail:smin5@snu.ac.kr

ORCID: https://orcid.org/0000-0002-6916-0489

*These authors contributed equally to this work as first authors.

(a) This is an open-access article distributed under the terms of the Creative Commons Attribution Non-Commercial License (http://creativecommons.org/ licenses/by-nc/4.0/), which permits unrestricted non-commercial use, distribution, and reproduction in any medium, provided the original work is properly cited. Copyright (C) 2021 The Korean Association of Oral and Maxillofacial Surgeons. All rights reserved.
}

tumors are mostly located intraosseous, can be found in both maxilla and mandible, preferentially located in the mandible. MOT is mostly found in the second and third decades ${ }^{3,7-9}$. Histopathologically, MOTs are characterized by fine stellate and spindle-shaped cells in a myxoid or mucoid extracellular matrix $^{10-12}$. The origin of MOT is thought to be derived from odontogenic ectomesenchyme of a developing tooth or undifferentiated mesenchymal cells in the periodontal ligament ${ }^{13}$. Panoramic radiograph and computed tomography (CT) image, MOTs mostly present as multilocular radiolucency with internal opaque trabeculations. The initial diagnosis can be made based on clinical features and imaging. Definite diagnosis is established by the biopsy.

Management of MOT varies depending on the location and size of the tumor, the patient's systemic condition, and the surgeon's experience. They range from minimally invasive excisional biopsies to a mass resection with reconstruction. Long-term follow-up is essential due to a significant high recurrence rate of MOTs. Recurrence rates for myxoma are up to $25 \%$ in the existing literature ${ }^{6}$.

We present three unusual cases of MOT including huge 
ameloblastic, ossifying fibromatic and myxomatous pattern, in the whole mandible of middle-aged man, in mandible and maxilla of a young woman. We also update and summarize the current treatment strategy of the MOT.

\section{Materials and Methods}

Patient data was reviewed and collected from the Electronic Medical Record system. From August 2002 to December 2018, three patients who diagnosed with MOT visited one surgeon in the Department of Oral and Maxillofacial Surgery at Seoul National Univesity Dental Hospital. Records for the total three patients was collected and analyzed clinically and radiologically. We herein report those cases with each patient's description and treatment procedures. This original study was approved by the Institutional Review Board of Seoul National University (No. S-D20200010).

\section{Results}

1. Case 1: Huge myxoma involving hemimandible in a middle-aged male

In 2002, a 48-year-old patient came to Seoul National University Dental Hospital outpatient clinic because of pain on the right mandibular, but then the follow-up was lost from 2003. At that time, the tumor mass already caused facial deformity and loss of mandibular teeth. In 2012, the patient came back with a huge mass in the mandibular region and was diagnosed with MOT. However, the patient did not follow the surgical treatment indication. Three years later, in 2015, the patient came to the hospital again with the will of treating. The patient had a huge swelling mass in whole mandibular, however, painless. During the extra-oral examination, palpation revealed a hard swelling unsmooth surface. The measured size of the tumor was $11.5 \mathrm{~cm} \times 8 \mathrm{~cm}$.(Fig. 1. D) Intra-oral examination revealed swelling oral mucosa and the vestibular space was obliterated.

A panoramic radiograph revealed a large multilocular expansile lesion with a tennis racket pattern.(Fig. 1. A) The severe swelling expanded and involved the alveolar ridge. There was a loss of teeth (only \#33 remained) and remained tooth related to the tumor. In the comparison of CT views between two time-points (2012 and 2015), the multilocular expansile lesion significantly increased in size. Large locule with low attenuation and fluid level, while the small locule was filled with soft tissue attenuation.(Fig. 1. B, 1. C) The CT analysis resulted in a conclusion of aggressive benign tumor, the differential diagnosis included MOT, ameloblastoma, central giant cell granuloma (CGCG). Enhanced CT showed
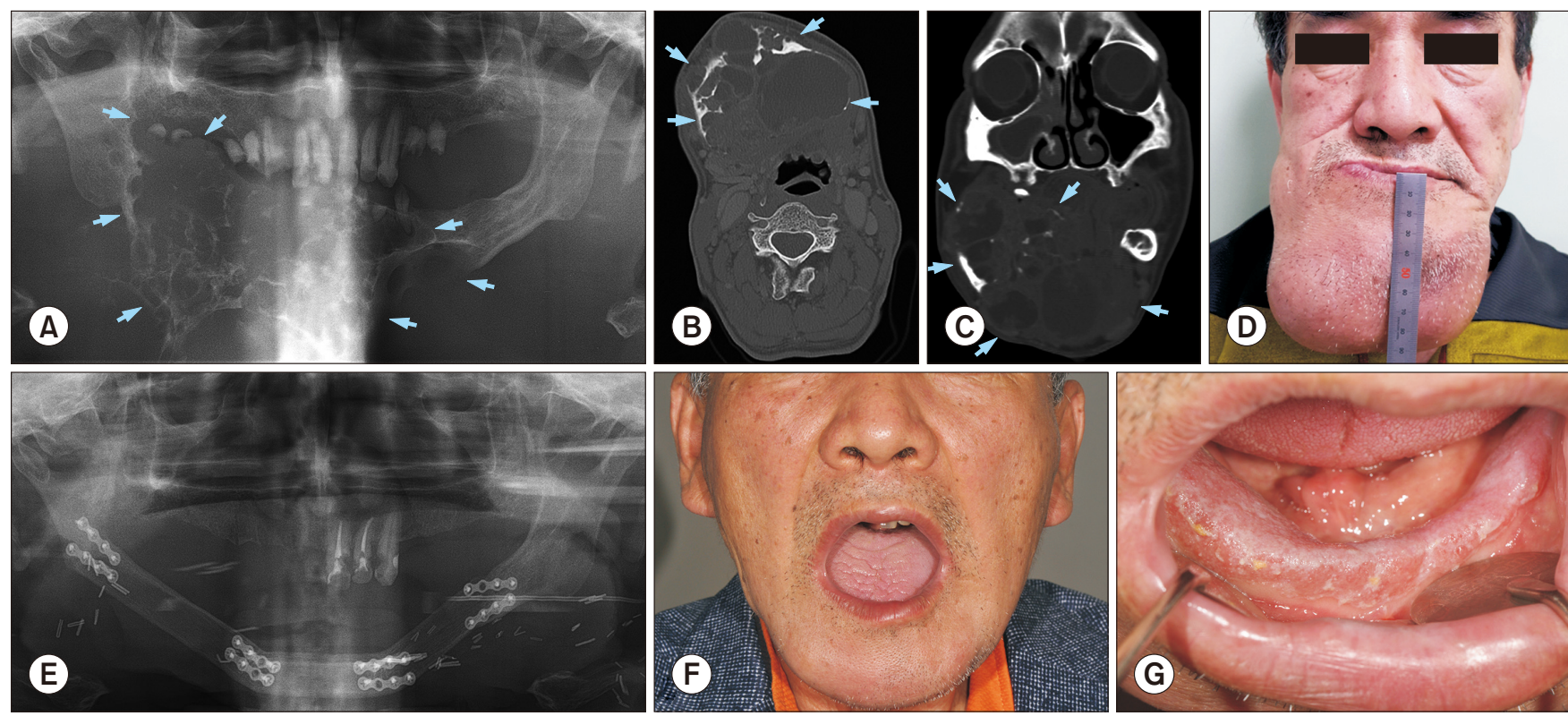

Fig. 1. Radiograms and clinical view of Case No. 1 with the giant myxomatous odontogenic tumor (arrows) in mandible. A. Preoperative panoramic view. B, C. Preoperative computed tomography image including axial and coronal views, respectively. D. Preoperative facial photo. E. Postoperative panoramic view, patient underwent mass resection and reconstruction surgery with fibular free flap. F. Extraoral view in 5-year follow-up visit, there was no sign of recurrence and patient achieve good mouth-opening. G. Intraoral view, 5-year follow-up. Truc Thi Hoang Nguyen et al: Large myxomatous odontogenic tumor in the jaw: a case series. J Korean Assoc Oral Maxillofac Surg 2021 
intrabony center and extrabone expansion of the tumor, adjacent to the growth of soft tissue. Right buccinator, masseter, mylohyoid, digastric, and geniohyoid muscles were expelled. A diffused fluid attenuation area was observed, and there was suspicious of hemorrhage presence. Enhanced CT suggested a multilocular benign tumor, mostly like MOT. Almost all indices related to red blood cells including hemoglobin ( $\mathrm{Hgb})$, hematocrit (Hct), and mean corpuscular volume (MCV) were decreased. However, there was increasing in platelet counting results. Besides, the erythrocyte sedimentation rate (ESR) index increased and the procalcitonin test showed the above normal level result.

The patient underwent mass excision, partial mandibulectomy and reconstruction with fibular free flap and splitthickness skin graft.(Fig. 1. E) Histopathological investigation using H\&E staining was performed, and the main biopsy has shown a loose myxomatous tumor cells filling the bony marrow spaces in the thin bony trabeculae. The reported result was MOT with ameloblastoma pattern and there was no metastasis at 14 regional lymph nodes. However, based on the clinical feature and radiological image, the final diagnosis was MOT. Immunohistochemical staining showed positive of CK-14, CK 18 , only a few positive of CK 7 and 5\% positive of $\mathrm{Ki}-67$.

During 4 years of follow-up visits, the patient showed excellent recovering with a good degree of mouth opening and a transformation of skin graft to the oral mucosa.(Fig. 1. F, 1. G)

\section{Case 2: Ossifying fibroma pattern in a young female}

A 16-year-old female patient came to Seoul National University Dental Hospital outpatient clinic with the chief complaint of extraoral and intraoral welling on the left mandibular posterior region. The swelling increased gradually and was painless. Medical history was non-contributory and there wasn't specific dental history either. During the extraoral examination, palpation revealed an ovoid, hard swelling in the left posterior mandible with the scabrous/unsmooth surface and indistinct borders. Intra-oral examination revealed a firm non-tender swelling. The borders of swelling mass were unclear, which extended from mesial proximity between \#35 and \#36 teeth to ramus and did not relate to the mandibular angle.

A panoramic radiograph revealed a large mixed radiolucent-radiopaque lesion with thinning, expansion and perforation of cortical bone, and radiating striae form of thin, wispy septa.(Fig. 2. A) The lamina dura of \#36, \#37 teeth were found mildly expanded, and \#38 tooth was observed relatively related to the tumor. The preliminary diagnosis derived from the panoramic view was a MOT or central hemangioma. CT axial and coronal image showed hypoattentuated mass in the mandible extending from mesial of \#36 tooth to left ramus region. Lingual and buccal cortical walls were found expanded and perforated. In addition, the relative between tooth \#38 and tumor mass could be observed on coronal images.

(Fig. 2. B, 2. C) Magnetic resonance imaging paranasal sinus (MRI PNS) showed the $4.5 \mathrm{~cm}$ size mass lesion with multiple septations with expansile nature and no evidence of significantly enlarged lymph nodes. MRI analysis results noticed a
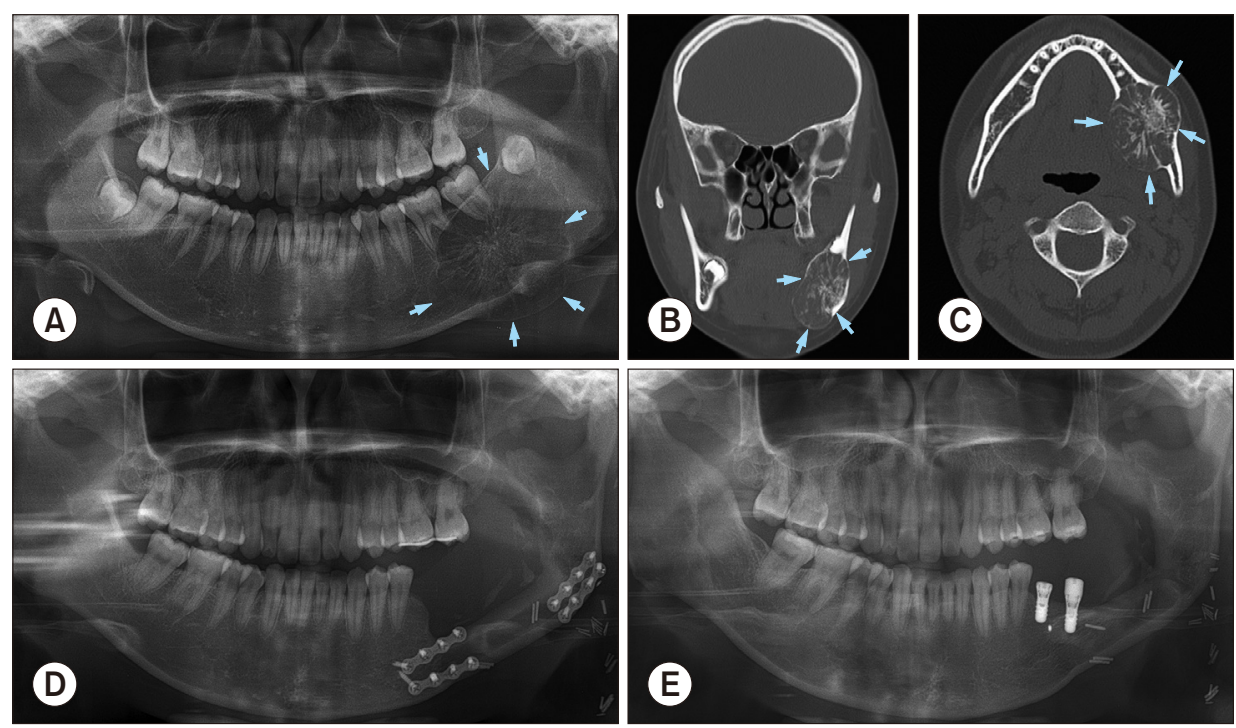

Fig. 2. Radiograms and clinical view of Case No. 2 with the large myxomatous odontogenic tumor (arrows) in mandible. A. Preoperative panoramic view. B, C. Preoperative computed tomography image including coronal and axial views. D. Postoperative panoramic view, the patient underwent resection and reconstructive surgery. E. Five years after odontogenic myxoma treatment surgery, bone augmentation and implant installation were performed.

Truc Thi Hoang Nguyen et al: Large myxomatous odontogenic tumor in the jaw: a case series. J Korean Assoc Oral Maxillofac Surg 2021 
low signal at T2-weighting and low, well enhancement at T1weighting, therefore the radiotherapist suggested a low possibility of myxoma. Whole-body fluorodeoxyglucose positron emission tomography examination showed a hypermetabolic mass on the left side mandible. Otherwise, no gross hypermetabolic lesion was noted to suggest distant metastasis in the rest of the imaged body. Evidence from the clinic and radiography suggested that this is a locally benign odontogenic tumor. The patient's white blood cell count was normal, however, the Hgb, Hct, MCV indices were decreased. The mean platelet volume (MPV) also decreased, along with increasing the ESR index. The patient also had an increase in the total protein result.

The patient underwent mass excision, surgical extraction of \#36, \#37, \#38 teeth and R-plate reconstruction surgery. Histopathological investigation using $\mathrm{H} \& \mathrm{E}$ staining was performed, and the reported result was MOT with the ossifying fibroma pattern. Nine months later, during follow-up care, R-plate was found fractured. Fractured plate removal and fibular free flap reconstructive surgery were indicated.(Fig. 2. D) Three years after fibular free flap reconstruction surgery, the defect region was reconstructed by block bone graft with the bone block harvested from left ramus and coronoid. The anterior plate was also removed during the grafting surgery. Another three years after bone augmentation surgery, posterior plate and micro screws were removed, and implant first operation at \#36, \#37 sites with consequent re-entry surgery was performed.(Fig. 2. E)

\section{Case 3: Involvement of unilateral whole maxilla}

A 36-year-old female patient had an asymptomatic slow swelling in the right maxillary region and came to Seoul National University Dental Hospital for examination in 2018. Medical history was non-contributory. The patient didn't smoke and had orthodontic treatment in 2010. A swelling mass could be observed on the radiograph view taken in 2016. It could be suggested that the tumor formed around the year 2016. Palpation revealed an ovoid, slight swelling in the right maxilla. The swelling had a smooth surface and indistinct borders. Intra-oral examination revealed an ovoid, nontender swelling. The swelling extended from the \#13 to \#17 tooth region with a smooth surface. The vestibular space was slightly obliterated.

Panoramic and Waters' radiographs revealed a mass in the maxilla and maxillary sinus.(Fig. 3. A, 3. B) The posterior wall of the sinus was perforated and mostly disappeared, the anterior wall was also indistinctive. The lesion extended from anterior to the posterior region of the maxilla and filled the whole right maxillary sinus. There was a displacement of \#15, \#16, \#17 teeth. CT image showed multilocular lesion that reached maxillary midline, filled the maxillary sinus. The
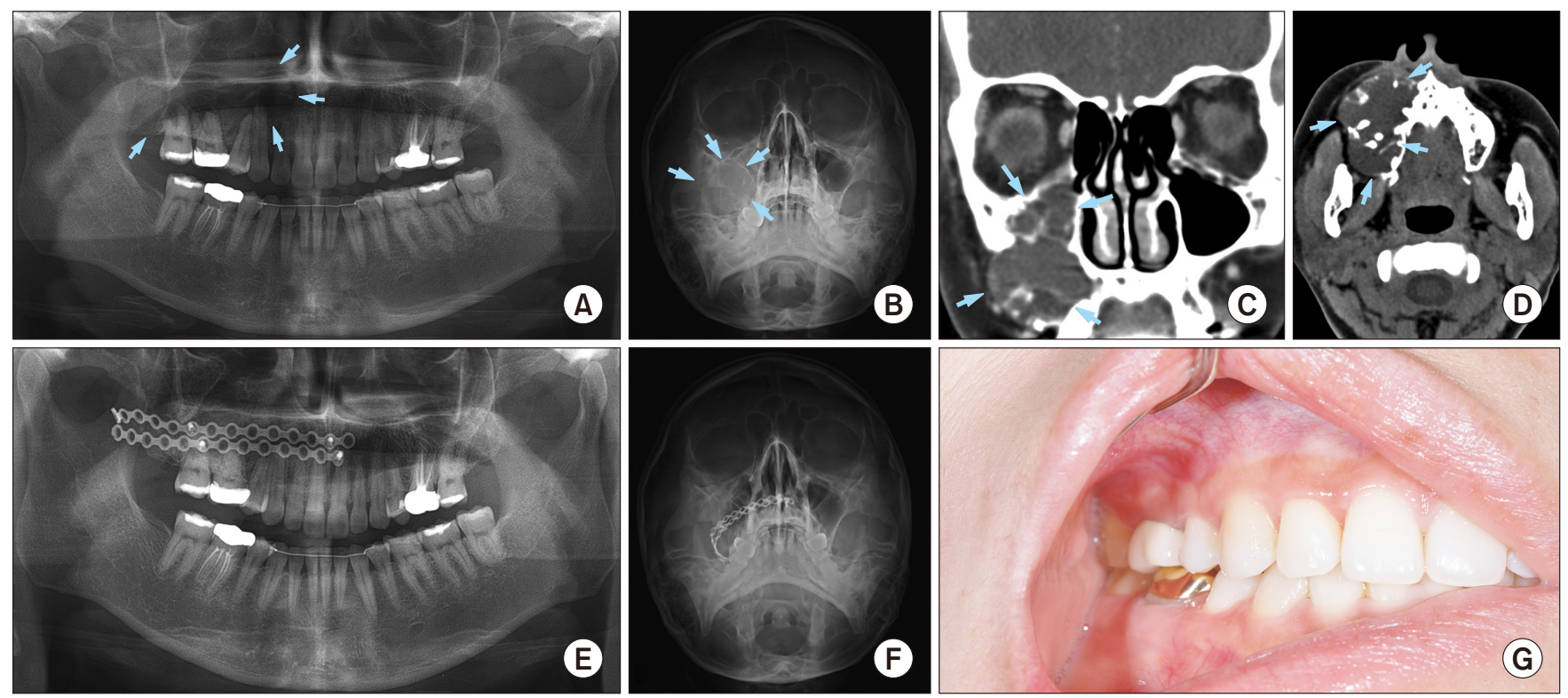

Fig. 3. Radiograms and clinical view of Case No. 3 with the large myxomatous odontogenic tumor (arrows) in mandible. A. Preoperative panoramic view. B. Preoperative Waters' view. C, D. Preoperative computed tomography image including coronal and axial views. E. Postoperative panoramic view, the patient underwent resection and reconstructive surgery with two plates. F. Postoperative Waters' view. G. Intraoral view at 1 -year follow-up visit.

Truc Thi Hoang Nguyen et al: Large myxomatous odontogenic tumor in the jaw: a case series. J Korean Assoc Oral Maxillofac Surg 2021 
posterior wall was swelling and perforated. The upper border of the tumor reached temporalis, posterior region of lateral and medial pterygoid muscle and approached pterygoid fissure.(Fig. 3. C, 3. D) The features on CT were suggestive of a benign tumor, such as dentinogenic ghost cell tumor or MOT, on the right maxilla. There were not many abnormalities in the patient's blood test result. MPV slightly increased and band neutrophil decreased.

Incision biopsy of submucosa at teeth \#16, \#17 regions showed the results of regenerating and remodeling trabecular bone and mucous material. The patient underwent mass excision, modified endoscopic sinus surgery (MESS), and twoplate reconstruction of the right maxillary sinus wall.(Fig. 3. E, 3. F) After surgery, the patient had slight hypoesthesia on the right ala of the nose and upper lip. Histopathological investigation using H\&E staining was performed, and the reported result was a MOT with OM pattern. After a one-year follow-up, the patient showed well recovery without hypoesthesia sensation. There was no sign of recurrence, no lesion at plate related mucosa and no disturbance in occlusion.(Fig. 3. G)

\section{Discussion}

MOT is defined as a benign but locally aggressive tumor of the jawbones. The most affected region in both jaws is the molar region, and tumors mostly appear in mandible $e^{9,14}$. There are some studies suggested a female predilection of MOT, whereas others have reported no sex predilection ${ }^{13,15}$. Although some authors report symptoms like pain and paresthesia $^{13,16}$, MOT is generally depicted as a slow-growing tumor with the potential to attain considerable size without noticeable signs and symptoms. MOT represents $0.5 \%$ to $20 \%$ of all odontogenic tumors and is the third most common odontogenic lesion after odontoma and ameloblastoma, and has a higher occurrence in the mandible (66.4\%) than in the maxilla $(33.6 \%)^{17}$. According to the $\mathrm{WHO}, \mathrm{OM}$ is classified as an odontogenic tumor derived from mesenchyme and/or odontogenic ectomesenchyme with or without odontogenic epithelium $^{13}$. Benign neoplasm can be grouped into two categories. The most common type is facial bone-derived, which in the early days was subdivided into true osteogenic myxoma and MOT. The latter type is soft tissue myxoma derived from perioral soft tissue, parotid gland, ear, and lar$\mathrm{ynx}^{3,17}$. The evidence for its odontogenic origin can be exhibited from high incidence in tooth-bearing areas of the maxilla and mandible, occurrence in young individuals, association with missing or unerupted teeth and presence of odontogenic epithelium.

MOT is usually found in the second and third decades but the age range in which the disease is found is wide. We reported a case at a young age (16 years old), and a case in older age (48 years old). The MOT in the maxillary related to the maxillary sinus and paranasal structures is needed to pay attention too, due to its potential of invasion into the important structures, and the difficulty in radical surgery. Due to varied radiographic presentation, it makes difficult to diagnose lesion based on radiographic features alone. Proper diagnosis requires clinical, histological and radiographic correlation. Barros et al. ${ }^{18}$ in 1969 proposed two stages of radiologic patterns, first stage osteoporotic appearance, with more prominent medullary spaces separated by thin septa of bone, and has varied presentations spider web or tennis racket appearances. The second stage is the destructive phase with the large expansion, loss of internal locules, and perforation of the cortical bone with invasion into surrounding soft tissues. The extension of the tumor into the antrum could be observed in the maxilla. Sometimes the "ometimes appearance presents, due to the peripheral margin of the septa arranged at right angles to the margin.

MOT grow slowly without any symptoms. Late-appearing symptoms may include pain, paresthesia, ulceration, and tooth mobility. MOT located in the midline is a rare incidence $^{19}$. One of our cases had a huge growth of myxoma due to the painless enlargement reaching a considerable size prior to being diagnosed. The asymptomatic growth also affected patients' willing for treatment. Our first patient waited more than 10 years before deciding to undergo surgical treatment. This delay resulted in a giant tumor mass and hemimandibular resection surgery. The radiographic differential diagnosis includes ameloblastoma, odontogenic fibroma, dentigerous cyst, CGCG, Langerhans cell histiocytosis ( $\mathrm{LCH})$, and fibrous dysplasia. While the MOTs mostly involve the alveolus ridge, the CGCG, hemangioma, and LCH tumors don't show the relation to alveolus ridge on radiography. A tennis racket pattern can be used to differentiate MOT from other tumors that involve the alveolus ridge. In addition, in the expansion stage, the MOTs usually have the fusiform expansion pattern, while the soap bubble pattern can be observed in solid ameloblastoma and glandular odontogenic cyst. Histopathologically, MOTs are characterized by the embedding of stellate and spindle-shaped cells in a richly myxoid extracellular matrix. Collagen is usually present scarcely, however higher amounts of collagen may be present in some cases, which are defined as myxofibroma ${ }^{11}$. 
The recommended treatment strategy for MOT remains controversial due to the lack of evidence-based surgical guidelines, mainly because MOT is a rare neoplasm and single-center prospective investigation is impossible ${ }^{20}$. Treatment planning depends greatly on the size of the lesion as indicated by the greatest measurable diameter. The high rate of recurrence is associated with the absence of tumor capsule, showing a tendency of the myxomatous tissue to infiltrate into small bone marrow spaces ("pseudo-malignant" invasion), causing bone destruction following incomplete removal with an average recurrence rate ranging from $10 \%$ to $33 \%$ depending on the type of treatment. Therefore, radical approach such as segmental resection with immediate reconstruction has been recommended in cases of larger tumors. In the first case, the huge tumor requires subtotal mandibulectomy which the $1.0 \mathrm{~cm}$ margin and fibular free flap reconstruction were performed. Even though this radical treatment caused the large defect, the reconstruction showed a satisfy- ing outcome with no sign of recurrence.

The conservative surgical treatment is enucleation with wide curettage of normal tissue or a generous amount of apparently intact tissue or a marginal resection of the mandi$\mathrm{ble}^{21}$. Although there is a tendency for a conservative surgical approach for children and a more radical approach for adults, Kansy et al. ${ }^{3}$ did not support this management strategy, primarily because enucleation exhibited similar recurrence rates to partial maxillectomies. The advantage of this approach is the preservation of vital structures and maintaining oral function, and it could be applied to recurrence after a simple surgery.

Although the standard treatment of MOT is still under controversy, we suggest a strategy flowchart of diagnosis and treatment for MOT as Fig. $4^{6,22,23}$, which is based on our clinical experience and review literature.

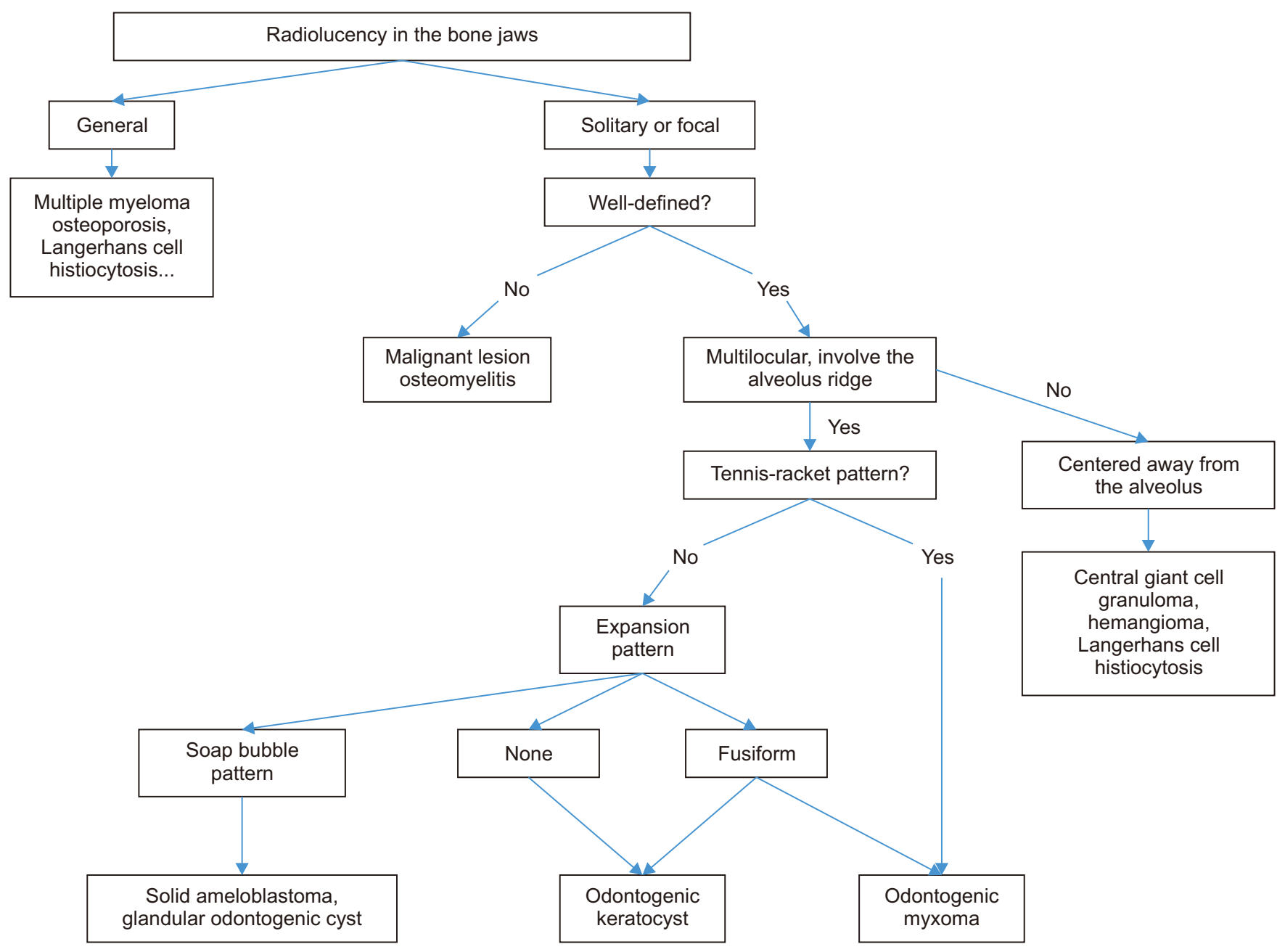

Fig. 4. A flowchart shows the diagnosis strategy of myxomatous odontogenic tumors.

Truc Thi Hoang Nguyen et al: Large myxomatous odontogenic tumor in the jaw: a case series. J Korean Assoc Oral Maxillofac Surg 2021 


\section{Conclusion}

Due to its slow-growing but locally invasive nature, MOT is usually detected accidentally or only when reaching a large size, causing bone expansion or even facial deformity. Surgical treatment depends on size, the position of the lesion, patient systemic condition and surgeon individual experience. In the case of a large tumor, radical resection and reconstruction is the standard surgical strategy. The conservative surgical treatment including enucleation with wide curettage is still under controversy. The recurrence rate for MOTs is significantly high, up to $30 \%$, therefore long-term follow-up is essential.

\section{ORCID}

Truc Thi Hoang Nguyen, https://orcid.org/0000-00028667-6698

Mi Young Eo, https://orcid.org/0000-0001-7055-9924

Yun Ju Cho, https://orcid.org/0000-0002-1818-5280

Hoon Myoung, https://orcid.org/0000-0002-9984-8479

Soung Min Kim, https://orcid.org/0000-0002-6916-0489

\section{Authors' Contributions}

T.T.H.N. and M.Y.E. participated in data collection and wrote the manuscript. Y.J.C participated in data collection and photographic editing. H.M. participated in the study design and data collection. S.M.K. participated in the study design and coordination and helped to draft the manuscript. All authors read and approved the final manuscript.

\section{Acknowledgements}

This research was supported by Basic Science Research Program through the National Research Foundation of Korea (NRF) funded by the Ministry of Education (No. 2017R1D1A1B04029339).

\section{Ethics Approval and Consent to Participate}

This original study was approved by the Institutional Review Board of Seoul National University (No. S-D20200010), and the written informed consent was obtained from all patients.

\section{Consent for Publishing Photographs}

Written informed consent was obtained from the patients for publication of this article and accompanying images.

\section{Conflict of Interest}

No potential conflict of interest relevant to this article was reported.

\section{References}

1. Kaffe I, Naor H, Buchner A. Clinical and radiological features of odontogenic myxoma of the jaws. Dentomaxillofac Radiol 1997;26:299-303. https://doi.org/10.1038/sj.dmfr.4600261

2. Buchner A, Merrell PW, Carpenter WM. Relative frequency of central odontogenic tumors: a study of 1,088 cases from Northern California and comparison to studies from other parts of the world. J Oral Maxillofac Surg 2006;64:1343-52. https://doi.org/10.1016/ j.joms.2006.05.019

3. Kansy K, Juergens P, Krol Z, Paulussen M, Baumhoer D, Bruder E, et al. Odontogenic myxoma: diagnostic and therapeutic challenges in paediatric and adult patients--a case series and review of the literature. J Craniomaxillofac Surg 2012;40:271-6. https://doi. org/10.1016/j.jcms.2011.04.009

4. Ochsenius G, Ortega A, Godoy L, Peñafiel C, Escobar E. Odontogenic tumors in Chile: a study of 362 cases. J Oral Pathol Med 2002;31:415-20. https://doi.org/10.1034/j.1600-0714.2002.00073.x

5. Thoma KH, Goldman HM. Central myxoma of the jaw. Oral Surg Oral Med Oral Pathol 1947;33:B532-40. https://doi. org/10.1016/0096-6347(47)90315-3

6. Wright JM, Soluk Tekkesin M. Odontogenic tumors: where are we in 2017? J Istanb Univ Fac Dent 2017;51(3 Suppl 1):S10-30. https://doi.org/10.17096/jiufd.52886

7. Mosqueda-Taylor A, Ledesma-Montes C, Caballero-Sandoval S, Portilla-Robertson J, Ruíz-Godoy Rivera LM, Meneses-García A. Odontogenic tumors in Mexico: a collaborative retrospective study of 349 cases. Oral Surg Oral Med Oral Pathol Oral Radiol Endod 1997;84:672-5. https://doi.org/10.1016/s1079-2104(97)90371-1

8. Mori M, Murakami M, Hirose I, Shimozato T. Histochemical studies of myxoma of the jaws. J Oral Surg 1975;33:529-36.

9. Noffke CE, Raubenheimer EJ, Chabikuli NJ, Bouckaert MM. Odontogenic myxoma: review of the literature and report of 30 cases from South Africa. Oral Surg Oral Med Oral Pathol Oral Radiol Endod 2007;104:101-9. https://doi.org/10.1016/ j.tripleo.2007.01.026

10. Barnes L, Eveson JW, Reichart P, Sidransky D. World Health Organization classification of tumours: pathology and genetics of head and neck tumours. Lyon: IARC Press; 2005.

11. Martínez-Mata G, Mosqueda-Taylor A, Carlos-Bregni R, de Almeida OP, Contreras-Vidaurre E, Vargas PA, et al. Odontogenic myxoma: clinico-pathological, immunohistochemical and ultrastructural findings of a multicentric series. Oral Oncol 2008;44:601-7. https:// doi.org/10.1016/j.oraloncology.2007.08.009

12. Li TJ, Sun LS, Luo HY. Odontogenic myxoma: a clinicopathologic study of 25 cases. Arch Pathol Lab Med 2006;130:1799-806.

13. Simon EN, Merkx MA, Vuhahula E, Ngassapa D, Stoelinga PJ. Odontogenic myxoma: a clinicopathological study of 33 cases. Int J Oral Maxillofac Surg 2004;33:333-7. https://doi.org/10.1016/ j.ijom.2003.12.004

14. Keszler A, Dominguez FV, Giannunzio G. Myxoma in childhood: an analysis of 10 cases. J Oral Maxillofac Surg 1995;53:518-21. 
https://doi.org/10.1016/0278-2391(95)90062-4

15. Adekeye EO, Avery BS, Edwards MB, Williams HK. Advanced central myxoma of the jaws in Nigeria. Clinical features, treatment and pathogenesis. Int J Oral Surg 1984;13:177-86. https://doi. org/10.1016/s0300-9785(84)80001-0

16. MacDonald-Jankowski DS, Yeung R, Lee KM, Li TK. Odontogenic myxomas in the Hong Kong Chinese: clinico-radiological presentation and systematic review. Dentomaxillofac Radiol 2002;31:71-83. https://doi.org/10.1038/sj.dmfr.4600678

17. Vijayabanu B, Sreeja C, Bharath N, Aesha I, Kannan VS, Devi M. Odontogenic myxoma of maxilla: a rare presentation in an elderly female. J Pharm Bioallied Sci 2015;7(Suppl 2):S759-62. https:// doi.org/10.4103/0975-7406.163550

18. Barros RE, Dominguez FV, Cabrini RL. Myxoma of the jaws. Oral Surg Oral Med Oral Pathol 1969;27:225-36. https://doi. org/10.1016/0030-4220(69)90177-7

19. Shivashankara C, Nidoni M, Patil S, Shashikala KT. Odontogenic myxoma: a review with report of an uncommon case with recurrence in the mandible of a teenage male. Saudi Dent J 2017;29:93101. https://doi.org/10.1016/j.sdentj.2017.02.003

20. Kauke M, Safi AF, Kreppel M, Grandoch A, Nickenig HJ, Zöller JE, et al. Size distribution and clinicoradiological signs of aggressiveness in odontogenic myxoma-three-dimensional analysis and systematic review. Dentomaxillofac Radiol 2018;47:20170262. https://doi.org/10.1259/dmfr.20170262

21. Mittal Y, Chugh A, Varghese KG, Dwivedi S, Goyal V. Management of recurrent odontogenic myxoma of mandible: a clinical case report. J Clin Diagn Res 2016;10:ZD30-1. https://doi.org/10.7860/ JCDR/2016/20917.8702

22. Dunfee BL, Sakai O, Pistey R, Gohel A. Radiologic and pathologic characteristics of benign and malignant lesions of the mandible. Radiographics 2006;26:1751-68. https://doi.org/10.1148/ rg.266055189

23. Chrcanovic BR, Gomez RS. Odontogenic myxoma: an updated analysis of 1,692 cases reported in the literature. Oral Dis 2019;25:676-83. https://doi.org/10.1111/odi.12875

How to cite this article: Nguyen TTH, Eo MY, Cho YJ, Myoung H, Kim SM. Large myxomatous odontogenic tumor in the jaw: a case series. J Korean Assoc Oral Maxillofac Surg 2021;47:112-119. https://doi.org/10.5125/jkaoms.2021.47.2.112 